\title{
Norois
}

Environnement, aménagement, société

249 | 2018

Patrimoine, vignoble, continuité écologique, transition énergétique, anthropologie des catastrophes

\section{Rayonner par la technique : des îles d'Outre-mer au cœur de la transition énergétique française?}

Excelling through technology: overseas territories at the heart of France's energy transition?

Sylvain Roche, Laurent Bellemare et Sylvie Ferrari

\section{OpenEdition}

Journals

\section{Édition électronique}

URL : https://journals.openedition.org/norois/7170

DOI : $10.4000 /$ norois. 7170

ISBN : 978-2-7535-7773-2

ISSN : $1760-8546$

Éditeur

Presses universitaires de Rennes

Édition imprimée

Date de publication : 31 décembre 2018

Pagination : 61-73

ISBN : 978-2-7535-7771-8

ISSN : 0029-182X

Référence électronique

Sylvain Roche, Laurent Bellemare et Sylvie Ferrari, «Rayonner par la technique : des îles d'Outre-mer au cœur de la transition énergétique française ? », Norois [En ligne], 249 | 2018, mis en ligne le 31 décembre 2020, consulté le 13 janvier 2022. URL : http://journals.openedition.org/norois/7170 ; DOI https://doi.org/10.4000/norois.7170 


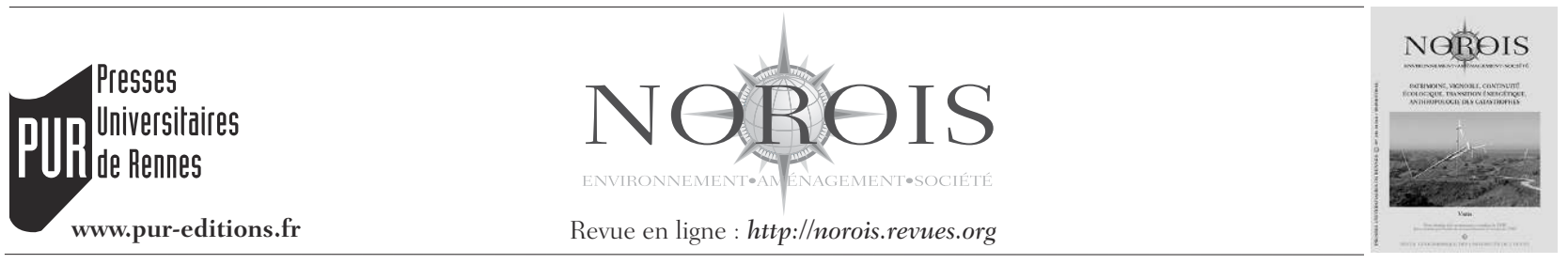

\title{
Rayonner par la technique : des îles d'Outre-mer au cœur de la transition énergétique française?
}

\author{
Excelling through Technology: Overseas Territories at the Heart of France's Energy Transition?
}

\author{
Sylvain Roche ${ }^{a}$, Laurent Bellemare ${ }^{\mathrm{b}}$ et Sylvie Ferrari ${ }^{\mathrm{a}}$
}

a GREThA, UMR CNRS 5113 , université de Bordeaux - 6 avenue Léon-Duguit, 33600 PESSAC.
(sylvain.roche@u-bordeaux.fr) (sylvie.ferrari@u-bordeaux.fr)
b Syndicat mixte d'Électricité de la Martinique, Fort-de-France. (bellemare.l@smem.fr)

Résumé : Mise à part la Guyane, les Outre-mer français sont des territoires insulaires de petite taille aux écosystèmes riches et sensibles ${ }^{1}$, soumis à la fois à de fortes pressions anthropiques et aux effets du changement climatique. Cette réalité encourage les pouvoirs publics à porter d'ambitieux programmes, en termes de développement durable, où la transition énergétique fait figure d'axe moteur. Isolés d'un point de vue énergétique et ne bénéficiant pas d'interconnexion aux réseaux électriques continentaux, ces « petits systèmes isolés » doivent trouver des solutions «à l'épreuve du climat » pour produire leur propre électricité, à partir des ressources naturelles valorisables, et satisfaire l'objectif d'autonomie énergétique à l'horizon 2030. L'article s'intéresse ici aux stratégies énergétiques déployées dans trois régions ultrapériphériques insulaires particulières : la Martinique, la Guadeloupe et la Réunion. Il interroge le rôle joué par les innovations technologiques dans ce processus de territorialisation marqué par des singularités multiples du fait de la variété des contextes géographique, historique, social et économique. En considérant les caractéristiques locales de la transition énergétique ainsi que le rôle joué par les différents acteurs (privés et publics), notre approche permet d’interroger la diversité des expériences régionales, en se focalisant sur les enjeux de production d'électricité renouvelable, dans les territoires insulaires considérés. Ainsi, elle fournit une grille de lecture originale des dynamiques de construction territoriale de la transition énergétique française.

\begin{abstract}
With the exception of French Guiana, France's overseas territories consist of small islands with rich and highly sensitive ecosystems that are subjected to strong anthropogenic pressures and the effects of climate change. This reality has encouraged public authorities to implement ambitious programs in terms of sustainable development where energy transition plays a crucial role. These "small isolated systems" - energy-insulated, with no interconnection to continental electricity networks - must find "climate-proof" solutions to produce their own electricity using recoverable natural resources, with the objective of achieving energy autonomy by 2030. This article focuses on the energy strategies deployed in three particular island territories: Martinique, Guadeloupe and Réunion. It questions the role played by technological innovation in this process of territorialization, which is characterised by multiple unique features owing to the variety of geographical, historical, social and economic contexts. By considering the local features of energy transition as well as the role played by various actors (both private and public), our approach examines the diversity of regional experiences by focusing on the issues and challenges of renewable electricity production in the island territories under study. In this way, it provides an original framework of interpretation regarding the dynamics of territorial construction at play in France's energy transition.
\end{abstract}

Mots clés : Énergie renouvelable - transition énergétique - innovation - gestion des ressources naturelles - insularité - France d'Outre-mer

Keywords: Renewable energy - energy transition - innovation - natural resources management - islands - French overseas territories

1. Classées parmi les 35 « hotspots » mondiaux, les îles des Caraïbes et en particulier les Antilles Françaises, bénéficient d’une biodiversité terrestre et marine exceptionnelle. 


\section{INTRODUCTION}

Les enjeux, liés à la crise énergétique et environnementale, ont bouleversé l'évolution des technologies de l'énergie en remettant en question les filières traditionnelles (énergies fossiles), tout en encourageant l'activation d'autres trajectoires technologiques. Le contexte d'environnementalisation des politiques énergétiques (Bouvier, 2012) a encouragé les îles à se positionner comme des territoires d'expérimentation des nouvelles technologies écologiques. Pour atteindre l'autosuffisance, les territoires d'Outre-mer misent sur leurs « avantages différenciatifs » (Pecqueur, 2006) en valorisant leurs ressources territoriales spécifiques (soleil, vent, géothermie, agro-énergie, énergies marines) qui s'avèrent être un atout considérable.

En tant que zones non interconnectées (ZNI), c'est-à-dire des territoires dont l'éloignement géographique empêche ou limite une connexion à un réseau d'envergure capable d'apporter certains services locaux (meilleure stabilité, approvisionnement en ressource primaire et achat d'énergie), les îles présentent des particularités géographiques qui demandent la mise en place de stratégies spécifiques. Tandis que les enjeux de lutte contre le changement climatique nécessitent de réaliser une véritable transition énergétique sur l'ensemble du territoire français, les problématiques ne sont pas les mêmes pour les îles d'Outre-mer. La géographie insulaire inscrit en effet la politique énergétique dans un système de contraintes à pallier ou à remédier (Nicolas, 2007). Leur isolement rend complexe leur approvisionnement énergétique et explique le choix souvent contraint de l'énergie thermique comme principale ressource. En cela les territoires d'Outre-mer ne diffèrent pas de la norme (hors pays nucléarisés ou bénéficiant de ressources hydrauliques importantes). Par contre, leur petite taille ne leur permet pas de bénéficier de l'économie d'échelle. Cette situation les oblige pour des raisons de sécurité à utiliser de nombreux équipements de faible puissance.

Plusieurs articles dans la presse nationale ont néanmoins attiré l'attention ces dernières années sur les expériences novatrices des Outre-mer dans le secteur de la transition énergétique : "L'outremer, laboratoire des énergies renouvelables ${ }^{1}$ », « La

1. La Croix, 22 mai 2017. [https://www.la-croix.com/Sciences-etethique/Environnement/Loutre-mer-laboratoire-energies-renouvelables-2017-05-22-1200849114].
Réunion, l'île de l'énergie verte ${ }^{2} »$, « La Martinique veut devenir un laboratoire de la transition énergétique $^{3}$ ", «La Réunion, laboratoire des énergies renouvelables ${ }^{4} »$. Les responsables locaux, particulièrement sensibles aux enjeux écologiques, tentent en effet depuis plusieurs années de décarboner l'énergie à la fois parce que c'est nécessaire, parce que les conditions climatiques s'y prêtent, et parce qu'une volonté politique forte les y encourage. Déjà en 1999, lors d'un colloque à l'Unesco, Paul Vergès alors Président du Conseil régional de la Réunion, fixait pour l'île l'objectif d'autonomie énergétique en électricité à l'horizon $2025^{5}$. De plus en plus la question de «l'après pétrole » se pose. Il est impossible, avec le pétrole, d'échapper à la volatilité des prix et de garantir l'accès à l'énergie, en cas de rupture prolongée d'approvisionnement. La question de la pollution et de la qualité de l'air intervient également, mais dans une moindre mesure.

La dimension territoriale du patrimoine naturel permet d'accorder une place centrale aux éléments de la nature (ressources naturelles et énergétiques, services écosystémiques associés aux systèmes environnementaux) dont la disponibilité peut contribuer au développement d'activités humaines spécifiques telle que la production d'énergie au sein de la société. La mise en concurrence, à l'échelle mondiale, des systèmes productifs incite en effet les petits territoires insulaires à se singulariser (Dehoorne, 2014) en mettant en avant des avantages différenciatifs spécifiques (Durand et al., 2015). Par définition, une « ressource constitue une réserve, un potentiel latent, voir virtuel, qui peut se transformer en actif si les conditions de production ou de création de technologie le permettent. » (Pecqueur, 2007 : 51). Les innovations technologiques deviennent ainsi des instruments de l'indépendance énergétique insulaire en valorisant des ressources naturelles spécifiques qui étaient jusqu'ici inexploitées. Devenus des laboratoires d'expérimentation à ciel ouvert, ces territoires préfigurent le réseau électrique français

2. Les Échos, 26 mai 2008. [https://www.lesechos.fr/26/05/2008/ LesEchos/20179-044-ECH_la-reunion--l-ile-de-l-energie-verte.htm].

3. La Gazette des Communes, 4 septembre 2014. [http://www.lagazettedescommunes.com/259817/la-martinique-veut-devenir-un-laboratoire-de-latransition-energetique/].

4. L'Usine Nouvelle, 9 septembre 2010. [https://www.usinenouvelle.com/ article/la-reunion-laboratoire-des-energies-renouvelables.N138013].

5. Témoignages, 31 mai 2008. [https://www.temoignages.re/developpement/energies/autonomie-energetique-impossible-n-est-pas-reunionnais, 30132]. 
de demain, où les énergies renouvelables auront vocation à remplacer les énergies fossiles dans le mix énergétique. Cependant la réalité des îles d'Outre-mer est large et variée de par les disparités existantes, dans des bassins géographiques aux caractéristiques spécifiques, mais aussi de par les disparités des échelles, tant en taille qu'en nombre d'habitants. Quand il s'agit d'énergies renouvelables, hormis pour le soleil, les ressources énergétiques dépendent du potentiel naturel des sites, là encore très variable selon les îles.

Si le concept de patrimoine naturel n'est pas nouveau, la préservation et la valorisation de l'environnement s'inscrivent aujourd'hui dans la perspective d'une gestion du territoire à long terme (Lefeuvre, 1990). La valorisation de certaines ressources naturelles ne peut alors être dissociée de leur préservation dans le temps, ce qui oriente nécessairement les choix des acteurs sur les territoires insulaires où elles sont produites, et transformées par le vecteur technologique. Le territoire est ainsi marqué par des relations étroites et spécifiques entre les caractéristiques environnementales et les caractéristiques techniques des ressources énergétiques dont il dispose, relations enchâssées dans des structures sociales et organisationnelles qui lui sont propres.

\section{LA FRAGILITÉ DU MODÈLE ÉNERGÉTIQUE INSULAIRE}

Les îles apparaissent comme des territoires contraignants du point de vue énergétique. Dépourvus de ressources fossiles et isolés au milieu des océans, les territoires insulaires ont pris conscience que leur avenir passait par le développement d'un nouveau modèle énergétique. Un rapport parlementaire de 2014 déposé par la commission des affaires économique indiquait que « les Outre-mer français ne peuvent continuer à présenter un bilan carbone de leur production électrique aussi dégradé. Seuls ces territoires continuent à être alimentés par des centrales thermiques au charbon et au fioul d'un autre âge. » (Bareigts et Fasquelle : 31). À titre d'exemple, le bilan carbone (ici contenu carbone du kWh électrique) s'élevait à $761 \mathrm{gCO}_{2} / \mathrm{kWh}$ par personne en Guadeloupe en 2015 contre $83 \mathrm{gCO}_{2} / \mathrm{kWh}$ dans l'Hexagone (OREC Guadeloupe, 2017 : 32). Ces chiffres sont néanmoins à nuancer du fait que ces territoires non nucléarisés n'auront pas à supporter les coûts prévus pour la dénucléarisation.

En prenant le cas de la Martinique, Yoann Pelis (2005) soulignait cette " pétro-dépendance » des îles caribéennes. En effet, contrairement aux premières stratégies de développement de l'électricité fondées sur l'hydroélectricité, le choix du pétrole comme source d'énergie pour la production d'électricité dans les années 1930 mit la Martinique dans une situation de dépendance extérieure au regard de l'approvisionnement des centrales électriques. Cette situation rendit l'île et, plus largement, les territoires d'Outre-mer vulnérables sur un plan environnemental mais aussi économique (Bayon, 2007). Cette particularité a deux conséquences : des tarifs énergétiques élevés et des risques de rupture d'approvisionnement plus importants. En 2016, la dépendance énergétique de la Martinique s'élevait à 93,75\% (OMEGA/AME, $2017: 13$ ) contre $46 \%$ pour la France en 2015 selon l'Office européen des statistiques ${ }^{6}$. Le coût de production moyen de l'électricité est estimé en 2013 à 259 €/MWh à la Martinique, contre environ 54,40 €/MWh en métropole (Le Gall et al., 2017 : 19).

Pour contrebalancer ces tarifs et lisser les prix de vente de l'électricité sur l'ensemble du territoire français (rôle de la péréquation tarifaire), les surcoûts de production des territoires d'Outre-mer sont pris en charge par la Contribution au service public de l'électricité (CSPE), un supplément payé par chaque consommateur sur sa facture. De cette façon, les consommateurs disposent d'une énergie au même prix que sur le reste du territoire national. Cette solidarité, nécessaire pour compenser le coût élevé de production de l'électricité dans les Outremer alors même que la consommation y est bien moindre, peut avoir des effets distributifs non seulement entre les consommateurs et les producteurs d'énergie, mais aussi parmi les secteurs d'activités qui utilisent l'énergie pour assurer leur production ${ }^{7}$. Ainsi, la mise en œuvre de la CSPE pour soutenir les énergies renouvelables conduit à faire supporter le coût des subventions par les consommateurs d'électricité tandis que parallèlement les industries

\footnotetext{
6. [https://www.touteleurope.eu/actualite/la-dependance-energetiqueeuropeenne.html].

7. Il faut noter ici que les industries les plus énergivores (ciment, aluminium...) bénéficient d'un régime dérogatoire (plafonnement de leur contribution au financement des énergies renouvelables) qui leur est favorable (exemption partielle ou totale de la CSPE).
} 
électro-intensives sont exemptées de la CSPE et ne paient pas un prix de l'électricité aussi élevé que les autres consommateurs. Une telle situation ne peut garantir la réduction de la consommation d'électricité dans l'ensemble de l'économie (Quirion, 2015).

Par ailleurs, il est important de noter que les ZNI ne contribuent en réalité que partiellement à cette CSPE dont la part la plus importante reste le financement des obligations d'achat (énergies renouvelables et cogénération) (figure 1).

La transition énergétique semble alors s'imposer comme une urgence d'autant plus que les ZNI disposent d'une certaine autonomie s'agissant de la politique de l'énergie depuis la loi du 13 décembre 2000 d'Orientation pour l'Outre-Mer (LOOM). Cette loi met en place une programmation pluriannuelle de l'énergie (PPE) par territoire, élaborée conjointement par le président de la collectivité et le préfet. Ce n'est seulement que depuis la Loi de Transition Énergétique pour la Croissance Verte de 2015 que la PPE fait intervenir les collectivités locales. Cette loi renforce les pouvoirs des collectivités en matière d'énergie et de développement durable. Auparavant EDF et l'État se chargeaient de cette PPE (qui s'appelait alors PPI pour Programmation Pluriannuelle des Investissements), élaborée principalement à partir des bilans prévi-

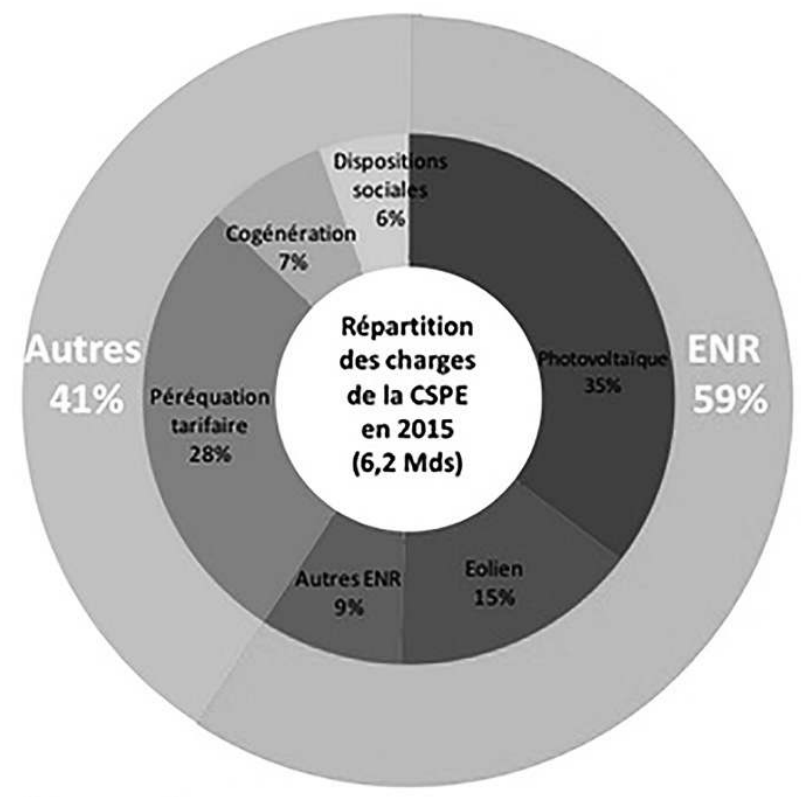

Figure 1 : Répartition des charges de la CSPE en 2015 (source Atlante, 2016)

Distribution of CSPE charges (tax contributions for public utilities) in 2015 sionnels des besoins établis par EDF. Des stratégies d'actions ont, dès lors, été lancées avec l'objectif de développer un nouveau modèle énergétique insulaire. La loi relative à la transition énergétique pour la croissance verte, votée le 17 août 2015, fixe des objectifs ambitieux pour les territoires ultramarins. Dans son article 203, elle prévoit l'élaboration de PPE spécifiques pour la Corse et les Outre-mer. Les départements d'Outre-mer doivent en effet viser l'autonomie énergétique en 2030 (en termes de production électrique), avec, dès 2020, un objectif de $30 \%$ d'énergies renouvelables à Mayotte et $50 \%$ à la Réunion ${ }^{8}$, en Martinique, en Guadeloupe et en Guyane, contre $23 \%$ dans l'Hexagone. Dans le cadre de son Plan de Transition Énergétique 2015-2030 (Gouvernement de la Polynésie française, 2015), la Polynésie française, constituée de 118 îles, ambitionne de produire $50 \%$ de son électricité à partir des EnR en 2020 et de porter cette part à $75 \%$ d'ici à 2030. Wallis-et-Futuna (12000 habitants) quant à elle vise à produire $50 \%$ de son électricité avec des énergies renouvelables d'ici 2030 et $100 \%$ en 2050 (Territoires des îles Wallis et Futuna, 2017). Plus des $90 \%$ de l'électricité produite sont aujourd'hui issus des énergies fossiles. Cette amélioration de l'autonomie énergétique doit permettre de garantir et de sécuriser l'approvisionnement en électricité des territoires, en particulier en cas de tension sur les marchés du pétrole.

Dès 2012, les communes du nord de la Martinique (Cap Nord Martinique) se sont saisies du plan climat-énergie comme d'un outil de développement territorial durable. La communauté de communes est ainsi devenue lauréate de l'appel à projets national « Territoires à énergie positive pour la croissance verte ", en février 2015. L'annonce, faite le 26 juin 2015, de convertir le site de l'ancienne usine de production d'électricité EDF de Bellefontaine en Parc d'activités «transition énergétique - innovation » témoigne de cette volonté des acteurs publics de promouvoir le développement des énergies vertes sur le territoire martiniquais ${ }^{9}$. À ce jour, 19 territoires d'Outre-mer ont été labélisés " territoires à énergie positive ${ }^{10} »$ (Guadeloupe : 6, Guyane : 4, Réunion : 5, Martinique : 3, Mayotte : 1).

8. La PPE de la Réunion a été publiée le 14 avril 2017 au Journal officiel.

9. [http://www.capnordmartinique.fr/actualite/lancement-du-parc-d-activites-transition-energetique-innovation].

10. Un territoire à énergie positive (Tepos) vise l'objectif de réduire ses besoins d'énergie au maximum, par la sobriété et l'efficacité énergé- 


\section{LA TECHNOLOGIE COMME OUTIL DE L'AUTONOMIE ÉNERGÉTIQUE DES ÎLES D'OUTRE-MER}

Pour Alain Gioda, « les Outre-mer français sont un terrain exceptionnel de développement de nouvelles solutions d'énergie car ils disposent d'un grand nombre d'atouts ${ }^{11} »$. Nous retiendrons ici quatre " avantages différenciatifs " au sens de Pecqueur (2007) propres aux territoires insulaires qui expliquent cette situation :

- les îles disposent de nombreuses ressources naturelles valorisables;

- la taille réduite des territoires est un facteur permettant l'expérimentation en vraie grandeur. Les réseaux sont limités en nombre de points desservis et en étendue géographique;

- la faiblesse de leur demande en énergie finale y favorise le développement de petits systèmes énergétiques (micro-production). L'absence d'industrie lourde limite la demande en énergie finale;

- l'isolement, par rapport aux grands réseaux de distribution d'énergie, les encourage à développer des solutions locales et innovantes.

L'absence du nucléaire peut être vue telle une faiblesse pour les insulaires mais aussi comme une force à exploiter. Les Outre-mer n'ont en effet pas de «dépendance au sentier» (path dependence) tel l'Hexagone autour du nucléaire (Finon, 2008). Empruntée aux sciences économiques (Dosi, 1982), la notion de path dependence s'est développée en science politique, à la fin des années 1980, pour souligner le poids des choix effectués dans le passé et celui des institutions politiques sur les décisions présentes (David, 1986). Contrairement à l'Hexagone, aucune centrale nucléaire n’a été construite dans les Outre-mer, et de ce fait, les territoires insulaires n'ont pas une contrainte du maintien d'une technologie installée, parfois moins efficace (Farinelli et al., 1999). Cette absence du nucléaire est donc une facilité mais c'est aussi un discours promotionnel revendicatif, les territoires se présentant comme les seuls territoires dénucléarisés de France. Elle est une force d'un point de vue sécuritaire et environnemental ainsi qu'un levier pour négocier des moyens financiers. En effet, la compa-

tiques, et de les couvrir par les énergies renouvelables locales ( $100 \%$ renouvelables et plus »). [www.territoires-energie-positive.fr/].

11. Entretien avec Alain Gioda, Chercheur, Montpellier, UMR 5569 Hydrosciences, IRD, UM/CC MSE, 12 juillet 2017. raison sur le long terme du coût de l'énergie entre les territoires et le continent, en incluant les coûts de dénucléarisation, relativise fortement le déséquilibre annoncé.

Cette situation encourage les îles à se lancer dans des stratégies de leapfroging (Fudenberg et al., 1983), littéralement de saut technologique. Une stratégie de leapfroging se caractérise par «the adoption of advanced or state-of-the-art technology in an application area where immediate prior technology has not been adopted. » (Fong, 2009 : 3707). Ces stratégies offrent des occasions de développer des technologies, directement en adéquation avec les contraintes propres aux pays insulaires, en valorisant les ressources locales sans reproduire les schémas des pays industrialisés continentaux, aujourd'hui soumis à de fortes controverses (Liotard, 2008). Cette politique de «saut technologique »se matérialise notamment par la mise en place d'une politique par les démonstrateurs et prototypes.

Les dernières évolutions technologiques permettent en effet d'imaginer des expérimentations techniquement innovantes que l'on pourrait regrouper sous le vocable de «smart islands » : gestionnaires d'énergies, compteurs communicants, capteurs, batteries intelligentes, etc. Le développement des technologies énergétiques renouvelables, avec le stockage, et les innovations dans les batteries pourraient en effet améliorer le rendement des machines et apporter une solution à leur principale contrainte, leur intermittence. C'est ce qu'a bien compris l'île de la Réunion, avec son projet PEGASE, une centrale photovoltaïque associée à un système de stockage par batterie sodium-souffre : ce projet, développé à Saint-André, contribue à la recherche permettant d'aller au-delà des $30 \%$ d'énergies intermittentes sur le réseau ${ }^{12}$.

Le projet des Mille installations de gestion énergétique dans les îles (Millener), mené par EDF, est une opération pilote qui a pour objectif de tester les réseaux électriques intelligents (smart grids) et de favoriser l'intégration des énergies renouvelables dans les systèmes énergétiques insulaires (SEI) français $^{13}$. Le Cirque de Mafate a ainsi été connecté au

12. GreenUnivers, Grâce au stockage, le réseau réunionnais va tolérer $32 \%$ d'électricité solaire en 2016 : [https://www.greenunivers.com/2016/02/ grace-au-stockage-le-reseau-reunionnais-va-tolerer-32-delectricitesolaire-en-2016-139541/].

13. [https:/www.ademe.fr/sites/default/files/assets/documents/75094_millener.pdf]. 
tout premier microgrid $100 \%$ solaire de la Réunion le 27 juillet $2017^{14}$. La généralisation des chauffeeaux solaires et l'installation croissante de panneaux photovoltaïque préfigurent ce que deviendront les bâtiments du futur : des mini-centrales électriques collectant in situ des énergies renouvelables. Ces réseaux intelligents pourraient constituer un puissant levier de l'action territoriale, spécialement au niveau opérationnel de la distribution, renouvelant ainsi les relations entre collectivités territoriales et gestionnaires des réseaux de distribution. Ils préfigurent les futurs schémas qui seront déployés y compris sur les grands réseaux continentaux qui devront s'adapter à la décentralisation massive de la production d'énergie, les consommateurs devenant également producteurs.

En Martinique, le démonstrateur Nemo, piloté par AKUO Energy et Naval Group, préfigure ainsi une centrale de production thermique des mers (centrale OTEC), pouvant alimenter, d'ici 2020, 35000 foyers (Roche, 2018). Ce procédé permet également de produire de l'eau douce ou du froid pour la climatisation par grande profondeur (SWAC) telle qu'elle est expérimentée à la Réunion, en Polynésie et en projet à la Martinique. Le Grenelle de l'environnement (2007) et ensuite le Grenelle de la mer (2009) amplifièrent la dynamique en identifiant les DOM-TOM comme des territoires propices à développer les énergies des océans. Le texte final du Grenelle de la mer ne manquait pas d'affirmer que « L'Outre-mer sera la vitrine technologique et le territoire d'expérimentation de la France en matière d'énergies marines renouvelables ». La publicisation de la transition énergétique des Outre-mer s'opère donc dans le cadre d'un système institutionnel régional, mais aussi dans celui d'un système qui reste fortement orienté par les directives nationales (voir les apports de La loi relative à la transition énergétique pour la croissance verte).

D'autres technologies innovantes sont en cours de développement telles la climatisation solaire ${ }^{15}$ et les expérimentations en matière d'agri-énergie autour de la bagasse ${ }^{16}$. Ces technologies pionnières ont pour objectif d'expérimenter, dans les conditions

14. [https:/www.edf.fr/edf/accueil-magazine/la-reunion-teste-l-hydrogenepour-stocker-l-energie-solaire].

15. Clicanoo, La Réunion invente la climatisation solaire, 3 décembre 2005. [https://www.clicanoo.re/Economie/Article/2005/12/03/1 La-Reunioninvente-la-climatisation-solaire 47167].

16. [http://agriculture.gouv.fr/valorisation-de-la-biomasse-en-guadeloupe-0]. réelles d'utilisation, des innovations encore au stade de la R\&D afin d'en évaluer la faisabilité, très en amont de l'ouverture de marchés (Moisan, 2011). Ces démonstrateurs permettent aux ingénieurs de disposer d'une technologie expérimentale pour anticiper l'évolution du coût du kilowattheure. Ceux-ci permettent aux industriels de mettre en place des actions de communication publicitaire bien en amont de la commercialisation. Néanmoins, ces stratégies de leapfroging restent des stratégies longues et coûteuses. Les îles (souvent les collectivités territoriales) doivent participer financièrement aux recherches et au développement des technologies. Les autorités ne le nient pas, l'intervention de l'argent public est indispensable malgré l'implication du secteur privé. De plus, cela reste un pari technologique risqué, car elles mettent en œuvre des technologies innovantes avec très peu de recul (Gallagher, 2006). On peut ici citer par exemple les mésaventures du projet houlomoteur CETO sur l'île de la Réunion ${ }^{17}$. Il y a donc nécessité de développer une propre expertise locale afin d'accompagner les décideurs locaux dans l'exercice de leurs nouvelles compétences (processus d'intelligence et de compétence territoriales). En effet, il est très difficile pour les élus de faire des choix face aux nombreux produits et projets qui leur sont proposés. C'est, en ce sens, que l'Agence Martiniquaise de l'Énergie a été créée en 2012.

\section{DES LABORATOIRES VITRINES DE LA TRANSITION ÉNERGÉTIQUE}

Cumulant les contraintes liées à l'insularité et la petitesse, ces territoires sont des laboratoires pour tester des nouvelles technologies énergétiques. Il n'est donc pas étonnant de voir émerger les îles comme l'échelle territoriale pertinente pour porter des initiatives novatrices dans le champ de la transition énergétique. En reprenant les mots de Marie-Christine Zelem (2015 : 13), « la transition énergétique viendra des territoires qui fonctionnent comme de véritables laboratoires d'initiatives ». Un avis du Conseil économique, social et environnemental indiquait que «l'Outre-mer doit être, désor-

17. Le Marin, Le cyclone Bejisa a endommagé un prototype houlomoteur à la Réunion, 24 janvier 2014. [http://www.lemarin.fr/articles/detail/items/ le-cyclone-bejisa-a-endommage-un-prototype-houlomoteur-a-la-reunion. html]. 
mais, clairement considéré comme un véritable laboratoire » pour la transition énergétique (Galenon, 2011 : 105). Dans son introduction aux actes du colloque «L'île laboratoire » tenu à l'université de Corse en juin 1997, Anne Meistersheim (1999) rappelait que l'île a toujours été un « lieu d'utopie et d'expérimentation » pour les scientifiques et les ingénieurs. C'est « un objet récurrent de l'écologique scientifique »(Pelletier, 2011 : 489), un « laboratoire réflexif exemplaire de la science écologique » (Pelletier, 2011 : 494). Avec les enjeux de la transition écologique, les îles deviennent de véritables territoires expérimentaux en matière de nouvelles technologiques énergétiques, des « laboratoires hors les murs où la mise au point se fait par et dans la pratique $^{18} »$. Elles « sont considérées aujourd'hui comme des laboratoires parfaits, à haute visibilité pour les nouvelles énergies » (Giralt et Payen, 2008 : 223). On assisterait ainsi « à un glissement progressif des représentations et de l'imaginaire insulaire » (Rosillette, 2017), qui sont caricaturalement celles d'un « ultime éden entre paradis touristique et sanctuaire naturel » (Dehoorne, 2014), «vers un discours aujourd'hui plus tourné vers l'innovation et les technologies de l'environnement ${ }^{19}{ }$. Confrontées à des problématiques énergétiques avant-gardistes, les îles permettent de mettre en œuvre des expérimentations concrètes où les résultats sont observables rapidement. Isolées et de petite taille, elles peuvent basculer plus rapidement qu'un autre territoire vers de nouvelles technologies, tout en permettant une démonstration à une échelle suffisamment grande pour faire école (rapidité des retours d'expérience) ${ }^{20}$.

Le réseau est plus court, plus facile à appréhender et à modéliser dans son intégralité. On peut y tester des technologies avant qu'elles arrivent dans l'Hexagone. Ces territoires préfigurent des problèmes qui interviendront plus tard sur les grands réseaux avec le développement des énergies renouvelables. Ainsi que l'indiquent François Bertrand et Elsa Richard, « en France, les territoires d'Outre-mer sont perçus selon une double approche, considérées à la

18. Entretien avec Yves Bouvier, Maître de conférences en histoire contemporaine, UMR 8138 - Sirice, Université Paris-Sorbonne, 24 août 2017. 19. Entretien avec Alain Gioda, 12 juillet 2017.

20. Voir ici la série documentaire «Les îles du futur. Pionnières de la transition énergétique "sur le site internet de la chaîne Arte. Grâce à 5 exemples (l'Islande, Samso, les Orcades, Madère et El Hierro), ces reportages nous présentent comment les îles s'inscrivent continuellement dans la rupture technologique en se présentant à leurs voisins continentaux tels les nouveaux modèles énergétiques de référence. fois comme réserve de savoir-faire et de solutions inédites d'adaptation pour la France hexagonale et comme relais aptes à diffuser les savoir-faire français et à exercer la solidarité envers d'autres territoires insulaires moins préparés» (2010: 5).

L'inauguration de la première centrale éolienne française avec stockage à la Guadeloupe en juin 2016 est ici un bon exemple de cette politique de reproductibilité technologique recherchée entre l'Outre-mer et l'Hexagone ${ }^{21}$. Nous pouvons par ailleurs noter que l'accélération des phénomènes naturels violents dans les îles, tels les tempêtes ou les cyclones, vont pousser les industriels à développer des technologies innovantes sur ces territoires (éoliennes aux mâts rabattables par exemple ${ }^{22}$ ) qui pourront potentiellement être commercialisées à l'échelle mondiale (figure 2).

Cela confirme la thèse que le changement climatique accélère le processus d'« innovation climatique » (Touzard, 2017) et tend à reconfigurer les « logiques spatiales de l'innovation » (Griset et Fernandez, 2007), notamment dans le champ de l'énergie.

Enfin, une motivation particulière et spécifique aux îles les conduit à montrer l'exemple à leurs voisins continentaux en se présentant comme les nouveaux modèles énergétiques de référence. L'Outre-

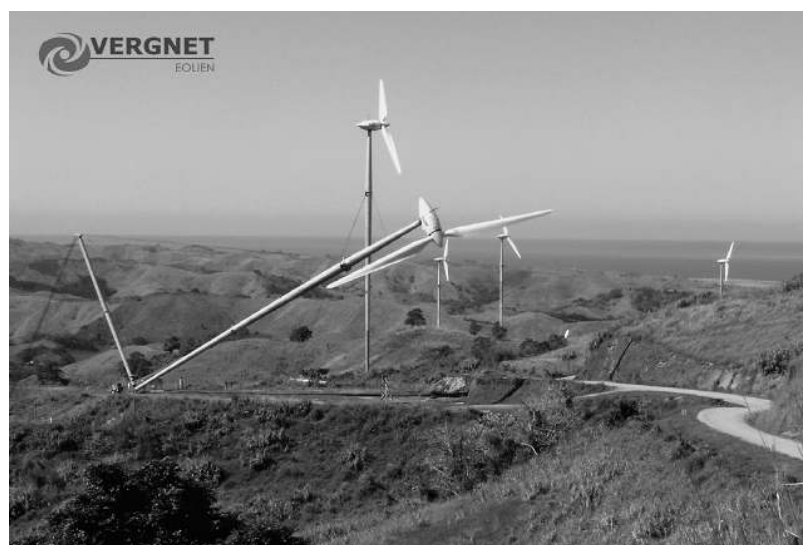

Figure 2 : Les éoliennes rabattables Vergnet ont résisté à l'ouragan IRMA (@ Vergnet) hurricane Irma Folding wind turbines produced by Vergnet withstood

21. Outremers360, Marie-Galante : Inauguration de la première centrale éolienne avec stockage, 30 juin 2016. [http://outremers360.com/economie/marie-galante-inauguration-de-la-premiere-centrale-eolienne-avecstockage/]

22. Le Monde de l'Énergie, Quand les éoliennes résistent à l'ouragan Irma, 14 septembre 2009. [https://www.lemondedelenergie.com/eoliennesvergnet-irma-ouragan/2017/09/13/]. 
mer se présente ainsi comme « vitrine technologique sur le monde » (ADEME, 2015 : 9), « une vitrine technologique de la France » (Stracom, 2008: 41) parce que certaines expériences menées dans ces territoires peuvent être adaptées à l'Hexagone et diffusées régionalement. Ces territoires constituent en effet des avant-postes français, et plus largement européens, au milieu de territoires non européens. En 2014, la ministre Ségolène Royal indiquait que les Outre-mer avaient « pour réussir cette mutation de pressantes raisons et de nombreux atouts. L'enjeu dès lors n'est plus de rattraper un vieux modèle à bout de souffle mais de devancer et d'entraîner ${ }^{23}$ » Il y a un effet «pilote régional » qui joue, les îles étant généralement des portions d'archipels où les solutions validées peuvent être généralisées facilement, avec des bénéfices économiques et politiques rapides (Pacifique, océan Indien, Caraïbes pour les îles françaises). «La Réunion est un petit laboratoire de tous les problèmes du monde » disait ainsi fièrement l'ancien Président du Conseil régional de la Réunion, Paul Vergès ${ }^{24}$. La transition énergétique offrirait ainsi un « rayonnement territorial » à ces territoires à travers la modernisation intellectuelle et technologique (Crusol, 2007a), les projets technologiques répondant à des objectifs politiques précis. Selon Louis Marrou et Nina Soulimant (2011), ces projets sont en effet souvent des vitrines pour un pays, une région ou une filière industrielle. Les bonnes pratiques développées en Martinique et en Guadeloupe, dans le secteur de l'énergie, peuvent essaimer dans les territoires alentour, d'influence anglo-saxonne et plus particulièrement états-unienne. Par exemple, la France a, jusqu'à 2015, fait office de pionnière en termes de géothermie, la Guadeloupe étant le seul territoire caribéen utilisant la géothermie pour produire de l'électricité. Cela a participé à mettre la France en position de challenger dans le développement commercial de cette filière industrielle ${ }^{25}$. À ce titre, nous pouvons dire que l'Hexagone souhaite profiter de

23. Déclaration de $\mathrm{M}^{\text {me }}$ Ségolène Royal, ministre de l'Écologie, du Développement durable et de l'Énergie, sur les engagements de la France en matière de protection de la biodiversité et de lutte contre le dérèglement climatique dans les Outre-mer, à Pointe-à-Pitre le 24 octobre 2014.

24. Discours de Paul Vergès lors de la $10^{\mathrm{e}}$ conférence des entreprises locales d'Outre-mer, à Saint-Denis de La Réunion le mercredi 19 novembre 2008.

25. Les Échos, La filière française de la géothermie se lance à l'assaut du marché mondial, 25 mars 2014. [https://www.lesechos.fr/25/03/2014/ LesEchos/2 1654-067-ECH_la-filiere-francaise-de-la-geothermie-selance-a-l-assaut-du-marche-mondial.htm]. «l'intérêt stratégique des insulaires ». Jean Crusol (2007b : 15) définit «l'intérêt stratégique des insulaires » comme "l'intérêt offert à une métropole par un territoire insulaire qui lui permet d'obtenir un avantage en matière de sécurité, de défense ou d'attaque militaire, en matière économique (proximité d'un gisement ou d'un marché, positionnement sur une route aérienne ou maritime...) ou symbolique (possibilité de rayonnement international...) ». Aujourd'hui le Canada, les États-Unis et de nombreux États européens cherchent à s'implanter dans ces territoires. La Chambre Franco-Allemande de Commerce et d'Industrie (CFACI) a d'ailleurs organisé depuis 2016 plusieurs séminaires afin d'intéresser les entreprises allemandes au marché caribéen. À l'heure où la spécialisation sectorielle des territoires est un enjeu majeur de compétitivité dans une économie mondialisée (Artus et Fontagné, 2006), ces projets technologiques territoriaux sont des marqueurs de différenciation par rapport aux autres régions, d'identification et de reconnaissance.

Dans cette optique, la Région Réunion a organisé une Conférence Internationale ClimatEnergie "Les îles et le changement climatique: Opportunités, Résilience, Adaptation »du 24 au 26 juin 2014 afin de mettre en avant sa politique volontariste en matière d'excellence énergétique et de lutte contre l'empreinte écologique ${ }^{26}$. Afin de poursuivre cette dynamique, notons que la Région Réunion organisait du 4 au 7 octobre 2017 en partenariat avec le Réseau de Gouvernements Régionaux pour le Développement Durable (nrg4SD), la deuxième édition de la Conférence internationale sur l'adaptation au changement climatique intitulée «Les îles, terres de solutions innovantes pour tous les territoires. » L'̂̂le se donnait alors comme objectif d'être « une référence pour d'autres régions, en particulier les territoires insulaires extrêmement vulnérables et les régions ultrapériphériques européennes (RUP), en termes d'autonomie énergétique et de solutions durables innovantes ${ }^{27}$ ».

En déplacement à la Martinique en septembre 2014, la ministre de l'Environnement, de l'Énergie et de la Mer, Ségolène Royal, déclara que «La Martinique peut devenir le laboratoire de ce

26. Franceinfo, Ouverture de la Conférence Climat-Energie à la Réunion, 24 juin 2014. [https://la lere.francetvinfo.fr/reunion/2014/06/24/ouverture-de-la-conference-climat-energie-la-reunion-163719.html].

27. Voir le programme de la Conférence. [http://www.nrg4sd.org/wp-content/ uploads/2015/06/ICProgram_FR.pdf]. 
que peut être le nouveau modèle énergétique français ». Comme l'indiquait le rapport Martinique, île durable territoire à énergie positive pour la croissance verte 2015-2020 : «L'insularité de la Martinique représente une opportunité inédite pour le déploiement des technologies françaises et européennes. Elle constituerait une vitrine de l'excellence européenne dans le bassin caribéen et pour les régions qui partagent les mêmes problématiques environnementales et socioéconomiques » (AME, 2015 : 4). La transition énergétique devient ainsi une «marque territoire» (Rochette, 2012) que les îles cherchent à vendre et à valoriser. Elle offre une tribune médiatique à des îles en quête de reconnaissance afin d'afficher leur réussite et leur dynamisme. Elle permet aussi aux territoires de bonifier et de communiquer sur leur offre territoriale de manière à attirer des investissements ainsi que des entreprises dans une démarche promotionnelle (HoullierGuibert, 2012).

De plus, il faut noter que l'enjeu est d'importance pour les grandes entreprises également qui ont besoin d'expérimenter sur ces territoires des technologies qu'ils devront déployer plus tard sur les grands réseaux continentaux. En ce sens, ces territoires constituent des pôles stratégiques clefs sur lesquels les acteurs de l'énergie cherchent à se positionner. Il y a deux concurrences : entre les territoires qui se positionnent et entre les compagnies qui cherchent à s'implanter. Cette dernière est toutefois plus récente mais elle tend à s'affirmer. Le marketing territorial prend donc une ampleur particulière dans l'agenda politique, du fait du caractère unique et novateur des projets. Cette communication se réalise sur un terreau favorable, à la croisée des politiques énergétiques et des politiques d'innovation.

\section{Conclusion}

L'examen de la trajectoire de trois îles d'Outremer a montré comment la transition énergétique s'inscrit dans des logiques territoriales qui lui sont propres, structurées selon les intérêts des acteurs et par des particularismes géographiques. En citant Nadaï et al. (2015 : 203) : « La mobilisation autour de projets s'opère en relation avec des enjeux de développement territorial, des héritages sociaux et spatiaux locaux. Les trajectoires innovantes sont donc toujours singulières. Elles ne peuvent être répliquées dans leur contenu ». L'exemple des îles d'Outre-mer nous oblige donc à porter un regard attentif sur l'histoire longue de ces territoires pour bien comprendre les trajectoires locales actuelles de transition énergétique.

Nul doute que le patrimoine naturel est un atout pour ces territoires. Soleil, vent, courants et houle, géothermie, énergie thermique des mers... Les idées ne manquent pas pour réussir la transition vers une société post-carbone. Et pourtant, le défi reste difficile pour un grand nombre de territoires. La transition demeure fragile. En effet, si certains territoires font figure d'exemples, d'autres peinent à exploiter leur potentiel et à mettre en place les solutions de leur transition énergétique. Entre 2012 et 2014, trois nouvelles unités fonctionnant au fioul sont entrées en service dans les territoires ultramarins : Port-Est à la Réunion, Bellefontaine en Martinique et Pointe Jarry en Guadeloupe. Cette situation est symbolique de ce verrouillage que continuent d'exercer les sources d'énergie fossiles sur les systèmes énergiques des Outre-mer (carbon lock-in). À titre d'exemple, le recours aux énergies fossiles pour produire l'électricité a ainsi augmenté de 1,3\% en Guadeloupe entre 2014 et 2015, la dépendance énergique n’ayant pratiquement pas diminué entre $2008(89 \%)$ et $2016(88 \%)$ (tableau 1).

La production de base s'appuyant sur le pétrole et le charbon est d'autant plus problématique que les prix des énergies fossiles restent encore aujourd'hui à des niveaux très bas et que l'évolution des prix reste soumise à de fortes controverses chez les économistes (ce qui participe à créer de l'incertitude) ${ }^{28}$. Des efforts supplémentaires doivent donc être réalisés bien au-delà de la production d'énergies vertes, qu'ils s'agissent des transports, de l'industrie, de l'agriculture ou du tourisme (Ceron et Dubois, 2012). À la Martinique en 2016, les carburants pour les transports représentaient plus de $60 \%$ de la consommation d'énergie finale tandis que la part des énergies renouvelables n'était que de 1,5\% (OMEGA/AME, 2017 : 29). L'année 2016 enregistrait à la Guadeloupe une augmentation de 1,9\% des consommations liées à l'électricité et de 3,8\% dans le secteur des transports, par rapport à 2015 (OREC Guadeloupe, 2017 : 2). On ne peut donc

28. Capital, Pourquoi le prix du pétrole a chuté et est-ce que ça va continuer? 10 mars 2017. 


\begin{tabular}{|c|c|c|c|c|c|c|}
\hline & \multicolumn{2}{|c|}{ Martinique } & \multicolumn{2}{|c|}{ Guadeloupe } & \multicolumn{2}{|c|}{ Réunion } \\
\hline & $\begin{array}{c}\text { Taux de } \\
\text { dépendance } \\
\text { énergétique \% }\end{array}$ & $\begin{array}{c}\% \text { d'électricité } \\
\text { d'origine } \\
\text { renouvelable }\end{array}$ & $\begin{array}{c}\text { Taux de } \\
\text { dépendance } \\
\text { énergétique \% }\end{array}$ & $\begin{array}{c}\% \text { d'électricité } \\
\text { d'origine } \\
\text { renouvelable }\end{array}$ & $\begin{array}{c}\text { Taux de } \\
\text { dépendance } \\
\text { énergétique \% }\end{array}$ & $\begin{array}{c}\% \text { d'ēlectricité } \\
\text { d'origine } \\
\text { renouvelable }\end{array}$ \\
\hline 2008 & 94,4 & 2,48 & 89,00 & 13,65 & 87,1 & 36,00 \\
\hline 2009 & 93,7 & 2,84 & 92,00 & 11,72 & 87,7 & 32,5 \\
\hline 2010 & 94,1 & 2,72 & 97,00 & 8,63 & 87,5 & 33,8 \\
\hline 2011 & 94,8 & 3,44 & 92,00 & 12,05 & 88,3 & 30,3 \\
\hline 2012 & 94,4 & 6,30 & 92,00 & 15,58 & 87,2 & 34,6 \\
\hline 2013 & 94,3 & 5,80 & 89,00 & 17,45 & 86,2 & 37,8 \\
\hline 2014 & 94,00 & 6,92 & 89,00 & 18,45 & 86,8 & 33,00 \\
\hline 2015 & 93.8 & 7,00 & 88,00 & 17,83 & 86,1 & 36,00 \\
\hline 2016 & 93,75 & 7,00 & 88,00 & 17,59 & 86,6 & 34,00 \\
\hline
\end{tabular}

Tableau 1 : Évolution de la situation énergétique de la Martinique, de la Guadeloupe et de la Réunion de 2008 à 2016 (sources : OMEGA/ AME, 2017; OREC Guadeloupe, 2017; SPL Énergies Réunion/OER, 2017)

Change in the energy situations of Martinique, Guadeloupe and Réunion between 2008 and 2016

pas parler de transition énergétique sans intégrer pleinement le transport dans la réflexion.

Dans ce contexte, la transition énergétique est un projet de territoire qui doit s'orienter vers des actions prioritaires bien ciblées et conduites dans la durée. Le fait que la transition énergétique, par son aspect innovant et populaire, soit portée comme un projet politique la met en danger. En Martinique, le secteur de l'énergie a été particulièrement impacté devant la volonté de faire table rase des initiatives précédentes. L'alternance politique associée à une refonte des institutions principales, à savoir la fusion de la Région et du Département en une nouvelle entité dénommée Collectivité Territoriale de la Martinique, a remis en cause les précédentes orientations à tous les niveaux. Les outils créés au cours de la mandature précédente ont été abandonnés, privant le territoire d'une expertise locale et de moyens d'action précieux (Société d'Économie Mixte Énergie de Martinique, Agence Martiniquaise de l'énergie (AME), SPL Martinique énergies nouvelles). Les élections de décembre 2015 en Martinique ont, de plus, stigmatisé l'opposition à la centrale biomasse Albioma ${ }^{29}$, puis au projet d'énergie thermique des mers $\mathrm{Nemo}^{30}$. La transition énergétique, appelée de leurs vœux par l'État et par les collectivités locales, se retrouve également entravée par ces mêmes institutions confrontées à des arbitrages économiques et politiques, motivés par des intérêts contradictoires voire contraires. C'est particulièrement prégnant si on se réfère à la récente démission du ministre de la Transition écologique et solidaire Nicolas Hulot, qui faisait part des contradictions au sein de l'État entre les discours et les actes, la volonté et les moyens, et surtout l'opposition fondamentale entre système libéral (croissance à tout prix) et sauvegarde de la planète.

Enfin, il convient d'insister que le fait que les questions du stockage d'énergie et de prévision de la ressource solaire et éolienne constituent des enjeux cruciaux pour le déploiement des énergies renouvelables en Outre-mer. Ainsi, il existe des limites dans

29. La biomasse est toujours rejetée par la mandature en place qui n'apparaît même plus dans la PPE (uniquement dans le volet validé avant la PPE) au bénéfice d'une filière d'éthanol improbable.

30. Le projet Nemo a été suspendu en avril 2018 pour des raisons liées à des difficultés techniques et des risques environnementaux. 
le développement des énergies intermittentes qui reposent sur la nécessité de garantir l'absence de blackout. Afin d'éviter de porter atteinte à la sûreté du système électrique, l'introduction de sources intermittentes d'énergie devrait rester en deçà du seuil de $30 \%$ (Mahiou, 2013) ${ }^{31}$. Cette situation est vraie pour le photovoltaïque sans stockage qui est par nature peu prévisible et intermittent. Le stockage d'énergie permet en effet de palier les incertitudes sur la ressource, de compenser la sousproduction, tandis que la prévision sur la ressource permet d'anticiper sur les moyens de production à mobiliser pour garantir l'équilibre offre/demande. L’Agence internationale de l'énergie (AIE) a publié le 6 juin 2017 son dernier rapport sur les perspectives technologiques en matière d'énergie. Elle y réaffirme clairement le rôle clé que peut jouer l'électricité décarbonée, associée à des solutions de stockage, pour atteindre les objectifs de lutte contre le changement climatique (AIE, 2017 : 62). Nous pensons que le déploiement des énergies renouvelables devrait donc s'intensifier avec le progrès technique dans le secteur des batteries, boosté par la conversion de l'industrie automobile à l'électricité, mais aussi via les autres formes de stockage telles que le thermique (froid/chaud), l'inertiel (volant d'inertie), le gravitaire (retenues collinaires, barrages et stations de transfert d'énergie par pompage) et l'hydrogène. Les grands acteurs de l'énergie (notamment EDF) développent actuellement des projets dans le secteur du stockage de grosses capacités centralisées (plusieurs mégawatts) et pilotées par le gestionnaire de réseau. La transition énergétique des Outre-mer se fera avant tout par la généralisation du concept de consommateur/producteur.

Les résultats en août 2017 de l'appel d'offres lancé en décembre 2016 pour la construction d'installations solaires en Corse et dans les départements d'Outre-mer ont montré l'intérêt économique du photovoltaïque avec stockage avec un prix moyen de 113,6 €/MWh. Ce prix est en baisse de plus de $40 \%$ par rapport à l'appel d'offres précédent de $2015^{32}$. Ces technologies entrent en effet en réelle compétition avec les centrales électriques traditionnelles,

31. Les experts ne sont néanmoins pas tous d'accord concernant ce seuil de puissance installée.

32. Communiqué de presse, Résultats de deux appels d'offres pour les installations de production d'électricité d'origine renouvelable en Corse et dans les départements d'Outre-mer, ministère de la Transition écologique et solidaire, Paris, 10 août 2017. tout en renforçant l'accès des particuliers (investissements privés) aux énergies renouvelables, qu’il s'agisse des panneaux solaires ou des éoliennes. C'est d'ailleurs ce qui est clairement prôné au niveau local : que la CSPE finance de l'investissement (CAPEX) plutôt que du fonctionnement (OPEX).

Notons enfin que cette dynamique d'innovations existe aussi dans l'Hexagone, où de petites communautés insulaires cherchent elles aussi à tirer des avantages économiques et sociaux issus du large potentiel énergétique de leur environnement ${ }^{33}$. Pour autant, la transition énergétique des îles françaises reste délicate du fait de la hiérarchie et de la structure politique même de l'État. Le modèle des petites îles écossaises ou scandinaves énergétiquement autonomes est difficilement reproductible en France. Ainsi le soulignait une responsable de l'Association des îles du Ponant : « Les îles d'Écosse ont un accès direct avec le gouvernement central, chose que nous n'avons pas en France. L'accès au pouvoir décisionnel des îles écossaises est beaucoup plus simple. En France, la hiérarchie bloque ${ }^{34} »$.

Une meilleure prise en compte des problématiques énergétiques insulaires semble néanmoins s'opérer aujourd'hui. Dans le cadre des débats autour du projet portant sur la loi Elan, le Sénat a adopté en juin 2018 deux amendements visant à autoriser la construction d'éoliennes dans les zones non interconnectées au réseau électrique métropolitain ${ }^{35}$. Cette mesure concerne plus spécifiquement les petites îles où l'implantation des machines est rendue difficile par la loi littoral. Pour le sénateur Michel Canevet : «Les îles sont les territoires les plus appropriés pour mener des expériences dans les énergies renouvelables. Elles doivent être exemplaires d'un point de vue énergétique. Or, en France, on a une surréglementation qui fait que c'est extrêmement difficile de mener des projets. Les contraintes administratives sont très dures, les procédures longues. Par exemple, la loi Littoral ne permet pas d'implanter des éoliennes en bordure du littoral et exige une distance minimale de 500 mètres entre les machines et les habitations. Sur

\footnotetext{
33. Certaines îles du Ponant, Molène, Ouessant et Sein, se sont fixé l'objectif d'atteindre l'autonomie énergétique dans une dizaine d'années. À Ouessant, il est prévu d'installer dans le Fromveur une ferme hydrolienne composée de deux à trois machines qui garantiront à l'île sa quasi autonomie énergétique.

34. Entretien avec Emilie Gauter, chargée de mission énergie à l'Association des îles du Ponant, 22 mai 2018.

35. [https://www.senat.fr/enseance/2017-2018/631/Amdt_336.html].
} 
les petites îles telles Ouessant ou Sein, la mer est partout et la loi Littoral s'applique partout. Il est donc impossible de mettre de l'éolien sur ces territoires dépendants du fioul. Ces amendements visent à débloquer cette situation ${ }^{36}$. »

Ainsi, la transition énergétique en milieu insulaire est de nature plurielle et il n'existe pas un modèle unique qui serait réplicable à d'autres territoires insulaires. La poursuite des objectifs de la loi de 2015 sur la transition énergétique pour la croissance verte se fera en fonction des contextes et des projets de développement qui sont propres aux îles d'Outre-mer. L'objectif de maîtrise de la consommation d'énergie devra être porté par une politique ambitieuse dans ce domaine en visant notamment les transports et l'habitat. Dans un contexte de croissance de la demande d'énergie, le développement de systèmes de production d'électricité renouvelable ne suffira pas. Des changements structurels dans les comportements sont nécessaires, changements s’inscrivant dans la sobriété énergétique.

\section{Bibliographie}

ADEME, 2015. Outre-mer, le grand potentiel, Magazine Ademe \& Vous, no 81, décembre 2014, janvier 2015, 16p.

AIE, 2017. Tracking Clean Energy Progress 2017, Energy Technology Perspectives 2017 Excerpt Informing Energy Sector Transformations, $116 \mathrm{p}$.

AME, 2015. Martinique, île durable, Territoire à énergie positive pour la croissance verte 2015-2020, $52 \mathrm{p}$.

Artus P., Fontagné L., 2006. Évolution récente du commerce extérieur français, Rapport du Conseil d'analyse économique, $\mathrm{n}^{\circ} 64,255 \mathrm{p}$.

ATLANTE, 2016. Réforme de la CSPE : les énergies carbonées au service de la transition énergétique, article du 15 janvier 2016. [http://atlante.fr/blog/reforme-de-la-cspe-les-energies-carbonees-au-service-de-la-transition-energetique/].

Bareigts E., Fasquelle D., 2014. Rapport d'information sur l'adaptation du droit de l'énergie aux Outre-mer, déposé par la commission des affaires économiques, enregistré à la Présidence de l'Assemblée nationale le 17 septembre 2014, Paris, $92 \mathrm{p}$.

Bayon D., 2007. Des économies vulnérables et dépendantes, in Levratto N. (éd.), Comprendre les économies d'outre-mer, Chapitre 2, Paris, L'Harmattan, p. 67-103.

Bertrand F., Richard E., 2010. Adaptation des territoires insulaires : éléments de réflexion à partir de deux îles françaises (Ré et La Réunion), VertigO - La revue électronique

36. Entretien avec Michel Canevet, sénateur du Finistère et porteur de l'un des amendements, 30 août 2018. en sciences de l'environnement, Vol. 10, n³ 3 , DOI : 10.4000/ vertigo. 10473

Bouvier Y. (dir.), 2012. Les défis énergétiques du XXI siècle. Transition, concurrence et efficacité au prisme des sciences humaines, P.I.E. Peter Lang, Bruxelles, p. 103-110

Ceron J.-P., Dubois G., 2012. Le tourisme dans l'outre-mer français face à la contrainte carbone, Mondes en développement, vol. 157, $\mathrm{n}^{\circ}$ 1, p. 11-28.

Crusol J., 2007(a). Les îles face aux enjeux du Xxi ${ }^{\mathrm{e}}$ siècle : statuts politiques, modernisation et capacité d'affronter la globalisation, in LAmbourdière E. (dir.), Les Caraïbes dans la géopolitique mondiale, Paris, Ellipses, p. 87-133.

Crusol J., 2007(b). Les ruptures politiques d'après-guerre : la bipolarisation du monde et les voies divergentes de décolonisation insulaire, in LAmbourdière E. (dir.), Les Caraïbes dans la géopolitique mondiale, Paris, Ellipses, p. 11-47.

DAVID P.A., 1986. Understanding the economics of QWERTY, in Parket W. N. (Ed.) Economic History and the Modern Economist, Oxford, Blackwell, p. 30-49.

Dehoorne O., 2014. Les petits territoires insulaires : positionnement et stratégies de développement, Études caribéennes [En ligne], n 27-28, mis en ligne le 14 août 2014, consulté le 28 août 2017. DOI : 10.4000/etudescaribeennes.7250

Dosi G., 1982. Technological paradigms and technological trajectories: A suggested interpretation, Research Policy, 11(3), p. 147-162.

Durand L., Pecqueur B., Senil N., 2015. La transition énergétique par la territorialisation, in SCARWELL H.-J., LEDUCQ D., Groux A. (dir.), Réussir la transition énergétique, Presses Universitaires du Septentrion, Lille, Collection Environnement et société, p. 29-36.

FARINELli U. et al., 1999. Energy as Tool for Sustainable for African, Caribbean and Pacific countries, New York, Development European Commission and UNDP, 160 p.

FinON D., 2008. Force et inertie de la politique nucléaire française. Une co-évolution de la technologie et des institutions, in Comité pour l'histoire économique de la France (dir.), État et Énergie, XIX $\mathrm{XX}^{e}$ siècle, Paris, La Documentation Française, p. 183-215.

Fudenberg D. et al., 1983. Preemption, Leapfrogging, and Competition in Patent Races, European Economic Review, 1983, vol. 22, issue 1, p. 3-31.

Fong M.W., 2009. Technology leapfrogging for developing countries. In Encyclopedia of Information Science and Technology, Khosrow-Pour, Mehdi Hershey, Pa, USA, Ed. Information Science Reference, p. 3707-3713.

GaLenon P., 2011. Les énergies renouvelables Outre-mer : laboratoire pour notre avenir, Les avis du Conseil Économique, Social Et Environnemental, Paris, Les éditions des Journaux Officiels, $114 \mathrm{p}$.

Gallagher K.S., 2006. Limits to leapfrogging in energy technologies? Evidence from the Chinese automobile industry, Energy Policy, v. 34(4), p. 383-394.

Giralt M., Payen A., 2008. Politiques énergétiques et insularité, in Nadia M., Guerassimoff G. (dir.), Îles et énergie : un paysage de contrastes, Paris, Presses Des Mines, coll. « Libres Opinions », p. 207-226. 
Gouvernement de la Polynésie française, 2015, Plan de transition énergétique 2015-2030, Conseil des ministres, 2 novembre 2015. [https://m.la lere.francetvinfo.fr/polynesie/sites/regions_outremer/files/assets/documents/plan_de_ transition_energetique_2015-2030.pdf].

Griset P., Fernandez A., 2007, « Les logiques spatiales de L'InNOVATION, $\mathrm{XIX}^{\mathrm{e}}-\mathrm{xx}^{\mathrm{e}}$ siècles », Histoire, économie E société, vol. 26 année, nº 2, 2007, p. 3-13.

Houllier-Guibert C.E., 2012. De la communication publique vers le marketing des territoires : approche microsociologique de la fabrication de l'image de marque, Gestion et management public, volume 1/2, (2), p. 35-49.

Le Gall O. et al., 2017. Revue de dépenses. Péréquation tarifaire de l'électricité avec les zones non interconnectées, Inspection générale des finances, 205 p.

LeFEuvre J.-C., 1990. De la protection de la nature à la gestion du patrimoine naturel, in Jeudy H. P. (dir.), Patrimoines en folie, Paris, Éditions de la Maison des Sciences de l'Homme, p. 29-75.

Liotard C., 2008. L'électricité dans les îles : vers une production durable?, dans Maizi N., Guerassimoff G. Îles et énergie : un paysage de contrastes, Paris, Presses des Mines, coll. « Libres Opinions », p. 143-170.

Mahiou B., 2013. "Gérer les énergies intermittentes pour la production d'électricité dans des îles ", Annales des Mines - Responsabilité et environnement, vol. 69, nº 1, p. 60-71.

Marrou L., Soulimant N., 2011. Baisse de la population et concept d'île renouvelable dans l'archipel des Açores, in TAgLioni F. (dir.), Insularité et développement durable, Marseille, IRD Éditions, coll. «Objectifs Suds », p. 107-129.

Meistersheim A., 1999. L'̂́le laboratoire, Ajaccio, Éd. Alain Piazzola, $459 \mathrm{p}$.

Moisan F., 2011. Du fonds démonstrateur aux investissements d'avenir : promouvoir une offre française dans le domaine des technologies vertes, Annales des Mines - Responsabilité et environnement, vol. 61, n 1, p. 109-115.

NADAÏ A. et al., 2015. Les territoires face à la transition énergétique, les politiques face à la transition par les territoires? in Laville B., Thiébault S., Euzen A., Quelles solutions face au changement climatique, Paris, CNRS Éditions, p. 196204.

Nicolas T., 2007. L'insularité aujourd'hui : entre mythes et réalités, Études caribéennes, nº 6, DOI : 10.4000/etudescaribeennes. 509
OMEGA/AME., 2017. Bilan énergétique Martinique 2016. Chiffres clés, 72 p.

OREC Guadeloupe, 2017. Bilan énergétique Guadeloupe 2016, 40 p.

Pecqueur B., 2006. Le tournant territorial de l'économie globale, Espaces et sociétés, no 124-125, vol. 1, p. 17-32.

Pecqueur B., 2007. L'économie territoriale : une autre analyse de la globalisation, L'Économie politique, $\mathrm{n}^{\circ} 33$, vol. 1, p. 41-52.

Pelis Y., 2005. Les nowveaux défis de l'énergie à la Martinique, Publibook, Paris, $150 \mathrm{p}$.

Pelletier P., 2011. Post face : l'île-laboratoire, le retour?, in TAglioni F. (dir.), Insularité et développement durable, Marseille, IRD Éditions, p. 489-504.

Quirion Ph., 2015. Quels soutiens aux énergies renouvelables électriques?, Revue française d'économie, 2015/4 (Volume XXX), p. 105-140. DOI 10.3917/rfe.154.0105.

Roche S., 2018. L'énergie thermique des mers dans les Outremer français : un enjeu stratégique de territoire?, Études caribéennes [En ligne], $1 \mid$ Juillet 2018, mis en ligne le 15 juillet 2018, consulté le 24 juillet 2018. [http://journals. openedition.org/etudescaribeennes/11971].

Rochette C., 2012. L'approche ressources et compétences comme clé de lecture du processus d'élaboration d'une ressource originale : la marque territoire, Gestion et management public, volume 1/1, no 1, p. 4-20.

Rosillette J., 2017. La Réunion : vers une autonomie énergétique? Le Monde de l'Énergie, 14 avril 2017. [https:/www. lemondedelenergie.com/la-reunion-vers-une-autonomieenergetique/2017/04/14/].

SPL Énergies Réunion/OER, 2017. Bilan énergétique Île de La Réunion 2016, Édition 2017, 10 p.

Stracom, 2008. Ministère de l'Intérieur, de l'Outre-Mer et des collectivités territoriales, novembre 2008.

Territoires des îles Wallis et Futuna, 2017. Programmation pluriannuelle de l'énergie pour les îles Wallis et Futuna 20162018/2019-2023, mars 2017, 42 p.

Touzard J.-M., 2017. Innover face au Changement ClimaTIQUE, Innovations, vol. 54, nº 3, p. 5-13.

Zelem M.-C., 2015. Réussir la transition énergétique. Quelles dynamiques de changement? in Scarwell H.-J., LeducQ D., Groux A. (dir.), Réussir la transition énergétique, Lille, Presses Universitaires du Septentrion, coll. «Environnement et société », p. 13-16. 